\title{
Vitamin D Deficiency and Keloids: Causal Factor or Bystander?
}

\author{
Hamideh Moravvej Hamed Memariani Mojtaba Memariani
}

Skin Research Center, Shahid Beheshti University of Medical Sciences, Tehran, Iran

\section{Dear Editor,}

Keloid lesions are generally regarded as benign fibroproliferative skin disorders unique to human beings that are characterized by unremitting inflammation and deposition of fibrous components in the extracellular matrix [1]. These lesions appear as firm, rubbery, variably pruritic, or tender growths that expand beyond the dimensions of the original injury and rarely regress. Keloids are thought to be age-dependent, occurring chiefly between the first and third decades of life. Both genders are equally afflicted by the keloid lesions. The disease is more common in populations with darker skin complexion, often exhibiting familiar pattern [2]. Notwithstanding the wide array of available therapeutic modalities, the management of keloids still remains enigmatic for physicians. Intralesional steroid injections, surgical excision, topical 5-fluorouracil, cryotherapy, laser therapy, radiotherapy, and silicon occlusive dressing, or any combination thereof, are among the mainstay of keloid treatment [3].

As a fat-soluble pro-hormone, vitamin D (VD) exists in two forms of ergocalciferol $\left(\mathrm{VD}_{2}\right)$ and cholecalciferol $\left(\mathrm{VD}_{3}\right)$. The former is present in plants and some fish, while the latter is mainly synthesized in the skin by way of sunlight exposure [4]. There is a mounting body of evidence that VD occupies a prominent role in bone remodelling, calcium-phosphorus metabolism, cell differentiation, and inhibition of both inflammation and fibro- sis [5]. VD status is traditionally appraised by measurement of the serum concentration of 25-hydroxyvitamin $\mathrm{D}[25(\mathrm{OH})-\mathrm{VD}]$, the major circulating form of VD in humans, which can be considered as a clinical indicator for nutritional vitamin $\mathrm{D}$ deficiency (VDD). The deficiency has been implicated in more than a dozen types of internal cancers, cardiometabolic disorders, muscle weakness, different infections, and auto-immune diseases [5]. From a dermatological viewpoint, there exists growing evidence to support the link between VDD and several skin disorders including, but not limited to, psoriasis, atopic dermatitis, vitiligo, Alibert-Bazin syndrome, systemic lupus erythematosus, lichen planus, systemic sclerosis, ichthyosis, pemphigus vulgaris, alopecia areata, polymorphic light eruption, and skin fibrosis [6].

One attractive hypothesis, first propounded by Cooke et al. [7], is that the increased predilection for hypertrophic scarring and hyperpigmentation in moderate-tohigh melanin containing skin is associated with the reduced levels of $\mathrm{VD}_{3}$ in the skin. It has also been postulated that $\mathrm{VD}_{3}$ supplementation may be beneficial in mitigating both inflammation and incidence of scar formation in genetically susceptible individuals. Exactly a decade ago, Zhang et al. [8] were the first to realize that 1,25-dihydroxyvitamin $\mathrm{D}_{3}\left[1,25(\mathrm{OH})_{2}-\mathrm{VD}_{3}\right]$ considerably hinders transforming growth factor- $\beta 1$-induced extracellular matrix production such as type I collagen as 
well as fibronectin in keloid fibroblasts (KFs) at both mRNA and protein levels in vitro. Likewise, exposure to VD3 $(20 \mathrm{ng} / \mathrm{mL})$ brought about a profound decline of type I collagen expression levels in KFs [9]. Another noteworthy finding concerning the $1,25(\mathrm{OH})_{2}-\mathrm{VD}_{3}$ treatment was the augmented expression of matrix metalloproteinase-9, a potent collagen breakdown inducer [8]. Though these results are interesting, further research is necessary to cast some light upon other possible mechanisms behind anti-fibrotic effects of VD on KFs.

With respect to KFs, a 4-day exposure to as little as 10 pmol L-1 of $1,25(\mathrm{OH})_{2}-\mathrm{VD}_{3}$ sufficed for significant decrement in cell proliferation ( $p<0.05 \mathrm{vs.} \mathrm{control)} \mathrm{in} \mathrm{vitro} \mathrm{[8].}$ Similar observations were made in a subsequent study in which $\mathrm{VD}_{3}$ decreased proliferation of cultured KFs in a dose-dependent manner [9]. Of note, $1,25(\mathrm{OH})_{2}-\mathrm{VD}_{3}$ dose-dependently downregulated the expression of proliferating cell nuclear antigen, a proliferation marker essential for DNA replication and repair [8]. These findings affirm the anti-proliferative effects of $\mathrm{VD}_{3}$ on KFs. Yet another facet of the cellular response of KFs to $\mathrm{VD}_{3}(20$ $\mathrm{ng} / \mathrm{mL}$ ) is the reduced expression of anti-apoptotic factor Bcl-2 together with substantial increment of caspase-3 levels after $24 \mathrm{~h}$ of treatment [8]. Hence, it seems that apoptosis is a likely mechanism through which $\mathrm{VD}_{3}$ reduces cell proliferation of KFs in vitro.

The biological activities of VD are known to be exerted by the nuclear vitamin $\mathrm{D}$ receptor (VDR)-mediated control of target genes. In a study undertaken by Hahn and Supp [1], there were significant differences in nuclear localization of VDR between normal skin and keloid scars $(p<0.001)$, as evinced by immunohistochemistry analysis. Another study afforded convincing evidence for significantly lower VDR expression in keloid patients than that of healthy controls $(p<0.0001)$ [10]. Such striking differences were also evident when comparing normal skin samples from White and Black donors [1]. The exact reason for differential VDR localization in light versus dark skin is unknown, but it could be attributed to variations in VD levels [1]. On the basis of these observations and other data, the conclusion was drawn that VDR may play a role in keloid pathology.

It is worthwhile to note that the gene encoding the VDR harbors several polymorphic regions. In this respect, the most frequent polymorphisms of the VDR gene are BsmI, FokI, TaqI, and ApaI [11]. A cohort study genotyped four diallelic polymorphisms of the VDR gene (FokI C > T, TaqI T > C, BsmI A > G, ApaI G > T) in an attempt to determine the risk of developing keloids [12]. The genotype and the allele frequencies of Fok I, BsmI, and ApaI were not substantially different between the patients and healthy individuals. However, the TaqI C > T polymorphism was found to be closely linked with the incidence of keloids. In particular, individuals carrying the CC genotype of TaqI showed a higher chance of developing keloid lesions while having a significantly lower mean circulating levels of $1,25(\mathrm{OH}) 2-\mathrm{VD}_{3}$ in comparison to carries of the TT and CT genotypes [12]. From these data, individuals with TaqI gene polymorphisms appear to be predisposed to keloid development.

There are several studies dealing directly with the relationship between VD deficiency and the development of keloids or hypertrophic scars [5, 12-14]. Based on VD level cut-off value of $16.1 \mathrm{ng} / \mathrm{mL}$, it was shown that patients with keloids had markedly lower mean serum $1,25(\mathrm{OH})_{2}-\mathrm{VD}_{3}$ levels as compared to normal healthy individuals [12]. Further evidence in favor of these findings was gleaned from a cross-sectional analytical survey in which lower serum levels of $25(\mathrm{OH})$-VD showed correlation with keloid severity [13]. In this context, $43.75 \%$ of the patients suffering from severe keloids had mean serum VD levels of $12.34 \pm 2.61 \mathrm{ng} / \mathrm{mL}$. In another work [5], a statistically significant association existed between hypertrophic scarring and VD levels $(p<0.05)$. In line with these findings, a recent study found the serum 25(OH)-VD and tissue VDR to be substantially lower in keloid patients than in healthy controls [14]. In addition, a substantial negative correlation between serum $25(\mathrm{OH})$ VD and duration of keloids was noted [14]. Although these data are interesting, further large-scales studies are warranted in order to elucidate the contributary role of VD deficiency in keloid development.

Recently, in a study enrolling 40 patients with keloid lesions, weekly intralesional injection of VD with a dose of $0.2 \mathrm{~mL}$ (200,000 IU) per $1 \mathrm{~cm}$ lesion was beneficial in improving keloid scars [15]. This finding suggests intralesional VD as a novel, safe, and effective option for future treatment of keloid lesions.

As hinted at above, it seems that the nutritional status of VD is linked with keloid development. Remarkably, certain VDR polymorphisms are thought to be associated with higher risk of keloid formation. Despite promising anti-fibrotic properties of VD, randomized clinical trials are required to assess prophylactic and therapeutic values of VD against keloids and hypertrophic scars. Meanwhile, VD optimal dosages and various routes of administration should be scrutinized in future studies. Last but not least, VD supplementation as an adjunct to the current therapies for keloids may be superior to each modality alone. 


\section{Key Message}

Vitamin D deficiency and certain genetic polymorphisms in the vitamin D receptor seem to be associated with keloid development.

\section{Statement of Ethics}

The authors have no ethical conflicts to disclose.

\section{Funding Sources}

No funding was given for this study.

\section{Author Contributions}

All authors have contributed equally to this work.

\section{Conflict of Interest Statement}

The authors declare no potential conflicts of interest.

\section{References}

1 Hahn JM, Supp DM. Abnormal expression of the vitamin D receptor in keloid scars. Burns. 2017 Nov;43(7):1506-15.

2 Kilmister EJ, Paterson C, Brasch HD, Davis $\mathrm{PF}, \mathrm{Tan}$ ST. The role of the renin-angiotensin system and vitamin D in keloid disorder-A review. Front Surg. 2019 Nov 26;6:67.

3 Memariani H, Memariani M, Moravvej $\mathrm{H}$, Shahidi-Dadras M. Emerging and novel therapies for keloids: A compendious review. Sultan Qaboos Univ Med J. 2021 Feb;21(1):e2233.

4 Kulie T, Groff A, Redmer J, Hounshell J, Schrager S. Vitamin D: an evidence-based review. J Am Board Fam Med. 2009;22(6):698706.

5 Ince B, Uyar I, Dadaci M. Effect of vitamin D deficiency on hypertrophic scarring. Dermatol Surg. 2019 Feb;45(2):274-9.

6 Kechichian E, Ezzedine K. Vitamin D and the skin: An update for dermatologists. Am J Clin Dermatol. 2018 Apr;19(2):223-35.
7 Cooke GL, Chien A, Brodsky A, Lee RC. Incidence of hypertrophic scars among African Americans linked to vitamin D-3 metabolism?. J Natl Med Assoc. 2005 Jul;97(7):10049.

8 Zhang GY, Cheng T, Luan Q, Liao T, Nie CL, Zheng $\mathrm{X}$, et al. Vitamin D: a novel therapeutic approach for keloid, an in vitro analysis. Br J Dermatol. 2011 Apr;164(4):729-37.

9 Mathangi Ramakrishnan K, Babu M, Lakshmi Madhavi MS. Response of keloid fibroblasts to Vitamin D3 and quercetin treatment - In vitro study. Ann Burns Fire Disasters. 2015 Sep 30;28:187-91.

10 Gong ZH, Ji JF, Yang J, Xiang T, Zhou CK, Pan XL, et al. Association of plasminogen activator inhibitor- 1 and vitamin $D$ receptor expression with the risk of keloid disease in a Chinese population. Kaohsiung J Med Sci. 2017 Jan;33(1):24-9.

11 Triantos C, Aggeletopoulou I, Kalafateli M, Spantidea PI, Vourli G, Diamantopoulou G, et al. Prognostic significance of vitamin D receptor (VDR) gene polymorphisms in liver cirrhosis. Sci Rep. 2018 Sep 14;8(1): 14065.

12 Yu D, Shang Y, Luo S, Hao L. The TaqI gene polymorphisms of VDR and the Circulating 1,25-Dihydroxyvitamin D levels confer the risk for the keloid scarring in Chinese cohorts. Cell Physiol Biochem. 2013;32(1):39-45.

13 Damanik VI, Putra IB, Ginting O. Correlation between serum 25-hydroxyvitamin $\mathrm{D}$ levels with keloid severity. Open Access Maced J Med Sci. 2019 Jan 12;7(1):65-7.

14 El Hadidi HH, Sobhi RM, Nada AM, AbdelGhaffar MMM, Shaker OG, El-Kalioby M. Does vitamin D deficiency predispose to keloids via dysregulation of koebnerisin (S100A15)? A case-control study. Wound Repair Regen. 2021 Jan 21.

15 Mamdouh M, Omar GA, Hafiz HAS, Ali SM. Role of vitamin D in treatment of keloid. J Cosmet Dermatol. 2021 Mar 15. 